23

\title{
Оптические характеристики наночастиц для эффективного ослабления излучения черного тела с температурой 2000-4000 градусов Кельвина
}

\author{
(С) В.К. Пустовалов ${ }^{1}$, Л.Г. Астафьева ${ }^{2}$ \\ ${ }^{1}$ Белорусский национальный технический университет, \\ 220013 Минск, Беларусь \\ ${ }^{2}$ Институт фозики им. Б.И. Степанова НАН Беларуси, \\ 220072 Минск, Беларусь \\ e-mail: astafev@dragon.bas-net.by
}

Поступила в редакцию 05.12.2019 г.

В окончательной редакции 05.12.2019 г.

Принята к публикации 16.12.2019 г.

\begin{abstract}
Исследованы возможности использования наночастиц для эффективного ослабления оптического излучения от различных природных или технологических источников (пожары, электрометаллургия, лазерная и плазменная обработка) с температурой поверхности излучателя в интервале $2000-4000 \mathrm{~K}$ на основе компьютерного моделирования. Излучение от вышеперечисленных источников может быть представлено как излучение черного тела с соответствующей температурой. Проведены исследования и анализ оптических характеристик двухслойных $\mathrm{SiO}_{2}-\mathrm{Au}-$ и $\mathrm{Fe}_{3} \mathrm{O}_{4}-\mathrm{Au}$-наночастиц систем ядро-оболочка в диапазоне радиусов 25-300 nm с толщиной оболочки 5 и $10 \mathrm{~nm}$ для длин волн излучения в спектральном интервале $200-500 \mathrm{~nm}$. $\mathrm{SiO}_{2}-\mathrm{Au}-$ и $\mathrm{Fe}_{3} \mathrm{O}_{4}-\mathrm{Au}$-наночастицы являются хорошими ослабителями (поглотителями) излучения с температурой $2000-4000 \mathrm{~K}$ в диапазоне оптического спектра $200-5000 \mathrm{~nm}$ и могут применяться в качестве возможных кандидатов для эффективного ослабления оптического излучения высокотемпературных источников.
\end{abstract}

Ключевые слова: двухслойные наночастицы, оптические свойства, излучение, анализ, ослабление.

DOI: $10.21883 /$ OS.2020.04.49209.331-19

\section{Введение}

В настоящей работе рассмотрены возможности использования наночастиц (НЧ) для ослабления интенсивного излучения от различных высокотемпературных источников. К числу таких источников излучения относятся пожары (включая горение нефти, газа, металлов) и технологические процессы, плазма и т.д. К числу высокотемпературных технологических процессов относятся электрометаллургия (дуговые печи), плазменная резка и сварка металлов, сварочные аппараты, лазерная обработка металлов и т.д. Все эти источники характеризуются рядом параметров - площадью поверхности высокотемпературного источника, температурой открытой поверхности излучателя, достигающей $2000-4000 \mathrm{~K}$, и другими факторами. Основными повреждающими факторами являются прямое воздействие области источника (пламени, плазмы) и дистанционное воздействие теплового излучения на предметы [1]. В результате воздействия интенсивного излучения при высоких температурах излучателя на предметы и поглощения его энергии предметы нагреваются, сжигаются и разрушаются. Люди умирают или получают ожоги тела и верхних дыхательных путей разной степени тяжести.
До 50\% тепла, поступающего от источника тепла, исходит от электромагнитного излучения [1-7]. Конвективный компонент менее значим. Окружающий воздух прозрачен (диатермичен) и несущественно ослабляет излучение, поэтому температура воздуха только немного повышается, когда излучение проходит через него.

Для зашиты от излучения используются различные способы. Целью применения защиты от воздействия интенсивного излучения является обеспечение безопасности и защиты жизни людей, а также защита или сведение к минимуму материального ущерба. Наиболее распространенным и эффективным способом защиты от теплового излучения является экранирование источника $[1,8]$. Экраны применяются для защиты от излучения источника, снижения облученности и температуры нагрева предметов, локализации тепловыделения в результате поглощения энергии излучения материалом экрана.

В прозрачных и полупрозрачных экранах излучение, взаимодействуя с веществом экрана, мало поглощается и распространяется внутри экрана по законам геометрической оптики, что обеспечивает видимость через экран. Так ведут себя экраны, выполненные из различных стекол: силикатного, кварцевого, металлизированного, а также из дисперсных водяных завес. В частности, огнестойкое стекло [9] обеспечивает защиту и пожарную безопасность жизни людей, защищает инфраструктуру 
здания, ограничивает ущерб от пожара и передачу теплового излучения до 30-60 min при определенной прозрачности в области видимого излучения.

Однако наряду с обеспечением прозрачности в видимой области спектра $(400-700 \mathrm{~nm})$ необходимо добиться значительного ослабления излучения в инфракрасной (ИК) области спектра (более $700 \mathrm{~nm}$ ), в которой концентрируется от 50 до 90\% энергии излучения в зависимости от температуры излучателя. В связи с этим необходимо улучшить ослабляющие свойства существующих экранов на основе стекол. Для этой цели в настоящей работе предлагается исследовать различные НЧ, размещенные в стекле, которые могут эффективно ослаблять энергию оптического излучения в ИК области спектра. Первым шагом в этом направлении является выбор и исследование оптических свойств отдельных НЧ.

В процессе воздействия оптического излучения на НЧ, размещенные в некоторой среде (стекле), происходит ослабление излучения. Ослабление излучения включает в себя эффекты поглощения и рассеяния излучения. Поглощение излучения происходит за счет поглощения НЧ и окружаюшей средой. С другой стороны, рассеяние излучения на НЧ и неоднородностях среды приводит к некоторому перераспределению интенсивности в среде в зависимости от характеристик излучения и самой среды, не вызывая существенного ослабления излучения средой. Максимальное ослабление оптической энергии будет реализовано в случае сильного поглощения (и преобразования энергии света в теплоту) и более слабого рассеяния излучения НЧ. Соотношение между поглощением и рассеянием излучения НЧ зависит от характеристик НЧ и самого излучения. Ранее было показано, что рассеяние излучения на отдельных НЧ существенно возрастает и даже становится больше, чем поглощение излучения НЧ, при размерах наночастиц больше $50-100 \mathrm{~nm}$ [10-14].

В последние годы в многочисленных работах были проведены исследования оптических свойств НЧ [10-24]. Поверхностный плазмонный резонанс излучения на НЧ определяет ее оптические свойства и эффективность поглощения и рассеяния излучения НЧ. Анализ оптических свойств различных однородных (металлических) НЧ показал практическую невозможность их использования для эффективного ослабления излучения в ИК спектральном интервале 700-5000 nm $[11,15,16]$. С другой стороны, среди различных НЧ выделяются сферические гибридные НЧ [12-14]. Сферические гибридные НЧ - это многослойные НЧ, состоящие из ядра и одного, двух или более сферических слоев, покрывающих ядро. Ядро и слои могут быть из разных материалов с разными геометрическими размерами. Среди НЧ из двухслойных систем ядро-оболочка можно упомянуть комбинации металл-металл, диэлектрикметалл и металл-диэлектрик, а также использование полупроводников, стекол в качестве материалов ядра и оболочки. Плазмонные свойства двухслойных НЧ зависят от их структуры, размера, формы, оптических свойств материалов ядра, оболочки и окружающей среды [12-14,17-22]. Изменяя размер ядра и толщину оболочки, плазмонный резонанс гибридной двухслойной НЧ можно смещать по длине волны в широком диапазоне оптического спектра.

Следует отметить, что НЧ используются в солнечных технологиях, основанных на поглощении солнечного излучения непрерывного спектра НЧ $[23,24]$. Но солнечное излучение имеет температуру поверхности Солнца около $5500 \mathrm{~K}$, и его максимальная интенсивность находится вблизи длины волны $550 \mathrm{~nm}$ видимого спектра оптического излучения. С другой стороны, излучение упомянутых выше оптических источников имеет температуру $T_{S}=2000-4000 \mathrm{~K}$, а их максимальная интенсивность находится в промежутке между длинами волн 700-1450 nm ИК спектра оптического излучения. Исследованные в [23,24] НЧ успешно использовались для поглощения солнечной радиации, но не могут быть использованы в целях эффективного ослабления излучения с температурами излучателя 2000-4000 K.

С другой стороны, сравнительное исследование и анализ оптимальных параметров различных двухслойных НЧ для использования их для эффективного ослабления излучения в широком спектральном интервале 200-6000 nm, составляющем 95\% спектра энергии излучения источников с температурой 2000-4000 К, в настоящее время отсутствует.

Существующий разрыв в знаниях между опубликованными результатами и потребностями в НЧ с соответствующими оптическими свойствами требует исследований и выбора оптических свойств, потенциально подходящих НЧ, которые могут быть применены для эффективного ослабления, поглощения и преобразования оптической энергии в системах защиты от излучения.

Основной целью нашей работы является исследование возможности использования выбранных НЧ для эффективного ослабления интенсивного оптического излучения от различных природных или технологических источников интенсивного излучения с температурой поверхности излучателя с интервалом 2000-4000 K на основе компьютерного моделирования. Объектом исследования являются спектрально-оптические характеристики одиночных двухслойных НЧ ядро-окисел и оболочка-металл, помещенных в стекло (кварц), в зависимости от длины волны излучения и параметров НЧ для эффективного ослабления (поглощения, рассеяния) оптического излучения ИК диапазона.

Отметим, что важно выбрать и исследовать НЧ с высокими температурами плавления и испарения материала ядра и поверхностного слоя двухслойной НЧ с целью ее сохранения в условиях воздействия интенсивного излучения, поглощения его энергии и нагрева НЧ. 


\section{Анализ оптических свойств двухслойных наночастиц окисел-металл}

Интенсивность теплового излучения зависит от температуры и площади источника, а также от степени черноты его поверхности. Излучение от всех вышеперечисленных источников излучения может быть смоделировано излучением черного тела (ЧТ) с достаточной степенью точности [1-8]. Модельную зависимость интенсивности оптического излучения $I_{\lambda}$ от длины волны $\lambda$ можно представить в виде спектра излучения ЧТ выражением [25]

$$
I_{\lambda} \sim \frac{8 \pi h c}{\lambda^{5}\left(\exp \left(h c / \lambda k T_{S}\right)-1\right)},
$$

$h$ и $k$ - постоянные Планка и Больцмана, $c-$ скорость света, $T_{S}$ - температура ЧТ.

Факторы эффективности поглощения $K_{\mathrm{abs}}$, рассеяния $K_{\text {sca }}$ и ослабления (экстинкции) $K_{\text {ext }}=K_{\text {abs }}+K_{\text {sca }}$ излучения с длиной волны $\lambda$ описывают оптические свойства сферической НЧ с радиусом $r_{0}$ [10]. Компьютерное моделирование $K_{\text {abs }}, K_{\text {sca }}$ и $K_{\text {ext }}$ различных двухслойных НЧ в зависимости от длины волны $\lambda$ в спектральном интервале 200-5000 nm и более было проведено на основе обобщенной теории Ми [10]. В уравнении (1) использовались следующие значения температуры ЧТ: $T_{S}=2000,3000,4000 \mathrm{~K}$. Энергия излучения для этих значений температуры ЧТ заключена в широком спектральном интервале $200-5000 \mathrm{~nm}$. Значения оптических показателей преломления и поглощения материалов НЧ (металлов, окислов) и окружающего стекла брались из [26,27]. Для оценки использования НЧ преимущественно в качестве абсорбера (поглотителя) или рассеивателя излучения использовался параметр

$$
P_{1}=\frac{K_{\mathrm{abs}}}{K_{\mathrm{sca}}}
$$

Все приведенные ниже рисунки описывают зависимости $K_{\text {abs }}, K_{\text {sca }}, K_{\text {ext }}$ и нормированной интенсивности излучения черного тела $I_{\lambda n}=\frac{I_{\lambda}(\lambda)}{I_{\lambda}\left(\lambda_{\max }\right)},\left(I_{\lambda}\right.$, деленной на максимальное значение интенсивности $I_{\lambda}\left(\lambda_{\max }\right)$, определяемой при значении длины волны $\left.\lambda_{\max }\right)$ для данного фиксированного значения $T_{S}$ от длины волны излучения $\lambda$ при фиксированных значениях радиуса НЧ и характеристик окружающей среды.

Отметим, что значение $\lambda_{\max }$ связано с температурой $T_{S}$ законом смещения Вина [25]

$$
\lambda_{\max } T_{S}=2.898 \cdot 10^{6} .
$$

Здесь $\lambda_{\max }$ измеряется в нанометрах, $T_{S}$ - в градуcax Кельвина. Длины волн $\lambda_{\max }$ для значений температуры $T_{S}=2000,3000,4000 \mathrm{~K}$ соответственно равны $\lambda_{\max }=1449,966,724.5 \mathrm{~nm}$.

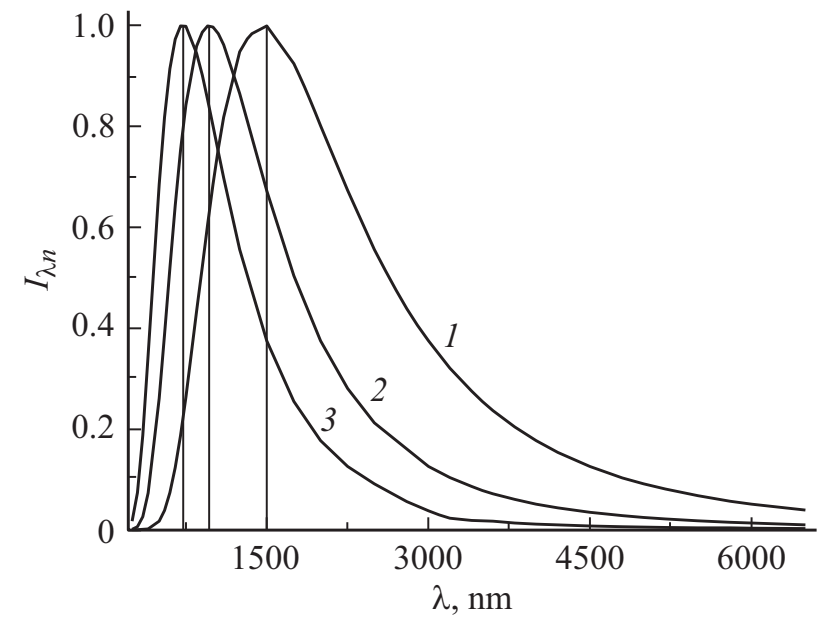

Рис. 1. Зависимость интенсивности $I_{\lambda n}$ оптического излучения ЧТ от длины волны $\lambda$ для трех значений его температуры: $T_{S}=2000(1), 3000(2), 4000 \mathrm{~K}(3)$.

На рис. 1 показано распределение нормированной интенсивности излучения ЧТ $I_{\lambda n}(\lambda)$ для трех значений температуры с $T_{S}=2000,3000,4000 \mathrm{~K}$ в соответствии c (1). Следует отметить, что длина волны $\lambda_{\max }$ в этом случае изменяется в интервале $\sim 700-1450 \mathrm{~nm}$ в ИК спектральном интервале.

Для эффективного ослабления излучения ЧТ в спектральном интервале 300-5000 nm необходимо, чтобы зависимость $K_{\text {ext }}(\lambda)$ была как можно ближе к зависимости $I_{\lambda n}(\lambda)$. Это связано с тем, что необходимо обеспечить ослабление всего спектра падающего излучения для каждой длины волны.

Основной целью нашего исследования является достижение максимального ослабления, поглощения излучения и минимального рассеяния при первом акте взаимодействия фотонов с НЧ для всего спектрального интервала излучения ЧТ. Эту ситуацию можно реализовать, если значение фактора ослабления будет максимальным $K_{\text {ext }}^{\max }$ и при этом параметр $P_{1}>1$ (или $\left.P_{1} \gg 1\right)$, т. е. фактор поглощения $K_{\text {abs }}$ должен быть больше (или намного больше), чем фактор рассеяния $K_{\text {sca }}$. Это означает, что максимальная часть энергии излучения (фотона) будет поглощаться НЧ, что позволяет достичь максимальной эффективности взаимодействия радиации с НЧ для ее ослабления. В противном случае, когда $K_{\text {sca }}>K_{\text {abs }}$ и $P_{1}<1$, рассеяние излучения НЧ будет доминировать над поглощением излучения НЧ, и в результате последующих актов взаимодействия фотонов с НЧ и многократного рассеяния произойдет перераспределение интенсивности излучения между НЧ и окружающей средой, и будет менее эффективное ослабление излучения, особенно при рассеянии излучения НЧ вперед. Этот случай является наименее интересным с точки зрения эффективности ослабления излучения.

Следовательно, для наших целей необходимо выбрать двухслойные НЧ, для которых $\lambda_{\mathrm{ext}}^{\max }$ находится 
вблизи $\lambda_{\max }$, соответствующей максимальному значению $K_{\mathrm{ext}}^{\max }$ в ИК области $\left(\lambda_{\max }\right.$ находится из уравнения (3) для излучения ЧТ с $\left.T_{S}=2000,3000,4000 \mathrm{~K}\right)$.

Предварительный анализ поглощающих и рассеивающих свойств различных НЧ ядро-оболочка [11-22] показал противоречивые и неудовлетворительные результаты для эффективного ослабления (поглощения) оптического излучения в широком диапазоне длин волн. Был проведен анализ оптических свойств широких классов НЧ с ядром металл, диэлектрик и оболочкой диэлектрик, металл (золото). Например, НЧ ядро-оболочка $\mathrm{Ni}-\mathrm{NiO}, \mathrm{Ti}_{-}-\mathrm{TiO}_{2}$ с различными значениями радиусов ядра $r_{0}$ и толщины оболочки $\Delta r_{1}$ не подходят для эффективного ослабление излучения ЧТ с температурами $T_{S}=2000-4000 \mathrm{~K}[24]$.

После предварительных расчетов и оценок были выбраны определенные двухслойные НЧ для возможного достижения эффективного ослабления излучений в ИК области. Оценки $\mathrm{HЧ}_{\mathrm{SiO}_{2}}-\mathrm{Au}$ и $\mathrm{Fe}_{3} \mathrm{O}_{4}-\mathrm{Au}$ показали возможность использовать их для эффективного ослабления излучения ЧТ с представленными температурами.

Рассмотрим оптические свойства НЧ для ослабления излучения ЧТ с температурой $T_{S}=2000,3000,4000 \mathrm{~K}$. Представляет интерес выбрать один тип НЧ с оптическими свойствами, которые позволяют использовать ее в качестве эффективного ослабителя (поглотителя) излучения во всем спектральном интервале оптического излучения при указанных температурах. По крайней мере, интересно выбрать НЧ с эффективным ослаблением излучения с какой-то одной температурой, например с $T_{S}=3000 \mathrm{~K}$, и с удовлетворительным ослаблением излучения с $T_{S}=2000,4000 \mathrm{~K}$. Это позволяет нам использовать одну НЧ для разных применений.

Для моделирования и анализа были выбраны двухслойные $\mathrm{HЧ} \mathrm{SiO}_{2}-\mathrm{Au}$ и $\mathrm{Fe}_{3} \mathrm{O}_{4}-\mathrm{Au}$ с толщиной оболочки $\Delta r_{1}=10 \mathrm{~nm}$ и радиусами НЧ в диапазоне $r_{1}=30-175 \mathrm{~nm}$, помещенные в $\mathrm{SiO}_{2}$.

Позиции $\lambda_{\max }$ и $\lambda_{\text {ext }}^{\max }$ расположения максимальных значений $I_{\lambda n}(T)$ и фактора эффективности $K_{\mathrm{ext}}^{\mathrm{max}}$ обозначены на рис. 2,3 разными вертикальными линиями - места расположения $\lambda_{\max }$ обозначаются сплошными линиями, места расположения $\lambda_{\text {ext }}^{\max }$ обозначаются штрихпунктирными линиями. Горизонтальные штриховые линии обозначают значение $P_{1}=1$ на рис. 2,3 .

На рис. 2 приведены зависимости $K_{\text {abs }}, K_{\text {sca }}, K_{\text {ext }}$ и $P_{1}$ сферических двухслойных $\mathrm{HЧ}^{\mathrm{SiO}_{2}}-\mathrm{Au}$ (с радиусами $r_{1}=30-170 \mathrm{~nm}$, толщиной оболочки $\Delta r_{1}=10 \mathrm{~nm}$ ) и оптической интенсивности $I_{\lambda n}$ ЧТ для $T_{S}=2000$, $3000,4000 \mathrm{~K}$ от длины волны $\lambda$.

Зависимости $K_{\text {ext }}\left(K_{\text {abs }}, K_{\text {sca }}\right)$ от длины волны $\lambda$ для представленных двухслойных НЧ имеют типичную форму плазмонных резонансов излучения на сферической НЧ ядро-оболочка в зависимости от значения радиуса $r_{1}$. Для значений $r_{1} \leq 70 \mathrm{~nm}$ в спектральном диапазоне 600-900 nm наблюдается резкая линия плазмонного резонанса с узкой полушириной на полувысоте, которая представляет практический интерес для нашего рассмотрения, несмотря на небольшую часть энергии излучения в этом интервале. С ростом значения $r_{1}>70 \mathrm{~nm}$ формируется второй плазмонный резонанс.

Для $r_{1} \geq 100 \mathrm{~nm}$ в спектральном диапазоне $1000-2000 \mathrm{~nm}$ наблюдаются 2 линии плазмонного резонанса с расположением двух максимумов факторов эффективности в ИК спектральном интервале. Для $r_{1}>130 \mathrm{~nm}$ происходит их объединение с большой полушириной, которая представляет практический интерес для нашего рассмотрения. Значения $K_{\text {ext }}$ Второго резонанса становятся больше, чем в предыдущем случае. При этом максимальные значения $K_{\mathrm{ext}}^{\max }$ достигают величин $K_{\mathrm{ext}}^{\max } \sim 8-10$ для $r_{1} \leq 70 \mathrm{~nm}$ и $K_{\mathrm{ext}}^{\max } \sim 4-6$ для $r_{1} \leq 170 \mathrm{~nm}$, что представляет значительный интерес.

Значения $r_{1}, \Delta r_{1}$ были выбраны на основе предварительного компьютерного моделирования таким образом, чтобы местоположения значений факторов $K_{\text {ext }}\left(K_{\text {abs }}, K_{\text {sca }}\right)$ могли быть смещены в широких пределах на оси $\lambda$. Значения $\lambda_{\text {ext }}^{\max }$ меньше $\lambda_{\max }$ на рис. $2,3, a, e, i$, они примерно равны на рис. $2, b, f, j$, и $\lambda_{\mathrm{ext}}^{\max }$ больше $\lambda_{\max }$ на рис. $2, c, g, k$. Таким образом, изменяя значения $r_{1}, \Delta r_{1}$ определенным образом, можно перемещать длину волны $\lambda_{\mathrm{ext}}^{\max }$ по оси $\lambda$, что позволяет подобрать оптические свойства НЧ для максимальной эффективности ослабления излучения ЧТ. Увеличение значения $r_{1}$ при толщине оболочки $\Delta r_{1}=10 \mathrm{~nm}$ приводит к сдвигу значения $\lambda_{\mathrm{ext}}^{\max }$ в сторону более длинных волн и соответственно к меньшим значениям температуры излучателя. Например, значение $\lambda_{\mathrm{ext}}^{\max }$ находится в точке $\lambda_{\mathrm{ext}}^{\max } \approx 810 \mathrm{~nm}$ при $r_{1}=60 \mathrm{~nm}$, что соответствует значению $T_{S} \approx 3580 \mathrm{~K}$, в точке $\lambda_{\text {ext }}^{\max } \approx 950 \mathrm{~nm}$ при $r_{1}=80 \mathrm{~nm}$, что соответствует значению $T_{S} \approx 3050 \mathrm{~K}$, и в точке $\lambda_{\mathrm{ext}}^{\max } \approx 1070 \mathrm{~nm} \mathrm{при}$ $r_{1}=100 \mathrm{~nm}$, что соответствует значению $T_{S} \approx 2708 \mathrm{~K}$ (рис. 2,e,f,g).

Зависимости $K_{\text {ext }}$ от $\lambda$ для НЧ $\mathrm{SiO}_{2}-\mathrm{Au} \mathrm{c} r_{1} \geq 100 \mathrm{~nm}$ для излучения с $T_{S}=2000$ и $3000 \mathrm{~K}$ соответственно близки к зависимости $I_{\lambda n}(\lambda)$ (на рис. 2, $c$ они практически перекрываются) в представленном спектральном диапазоне.

Значения параметра $P_{1}>1$ для $r_{1}=30-70 \mathrm{~nm}$, они достигают значений $P_{1} \sim 10-30$ и более для длин волн в диапазоне больше $2000 \mathrm{~nm}$ для температуры $T_{S}=4000 \mathrm{~K}$. Это означает, что поглощение излучения преобладает над рассеянием, и эти НЧ являются хорошими поглотителями (ослабителями) и плохими рассеивателями излучения. С ростом $r_{1}$ значения параметра $P_{1}$ становятся меньше 1 в спектральных интервалах $500<\lambda<2000 \mathrm{~nm}$ (для $r_{1}=100 \mathrm{~nm}$ ) и в интервалах $500<\lambda<4000 \mathrm{~nm}$ (для $r_{1} \sim 160 \mathrm{~nm}$ ) в зависимости от значения $T_{S}$, и рассеяние преобладает над поглощением.

Это означает, что НЧ $\mathrm{SiO}_{2}-\mathrm{Au}$ с некоторыми значениями $r_{1}, \Delta r_{1}$, например с $r_{1} \approx 60-100 \mathrm{~nm} \mathrm{и} \Delta r_{1}=10 \mathrm{~nm}$, является хорошим ослабителем из-за достаточного поглощения и в меньшей степени вследствие рассеяния излучения. Эффективное ослабление излучения в данной ситуации достигается при превышении поглощения над рассеянием. 


\begin{tabular}{|c|c|c|}
\hline \multicolumn{3}{|c|}{$\mathrm{SiO}_{2}-\mathrm{Au}, \Delta r_{1}=10 \mathrm{~nm}$} \\
\hline$T=2000 \mathrm{~K}, \lambda_{\max }=1450 \mathrm{~nm}$ & $T=3000 \mathrm{~K}, \lambda_{\max }=966 \mathrm{~nm}$ & $T=4000 \mathrm{~K}, \lambda_{\max }=724.5 \mathrm{~nm}$ \\
\hline$r_{1}=130 \mathrm{~nm}, \lambda \max _{\mathrm{ext}}=1260 \mathrm{~nm}$ & $=\frac{1000 \underset{\lambda, 2000}{0.8}}{r_{1}=60 \mathrm{~nm}, \lambda_{\mathrm{ext}}^{\max }=810 \mathrm{~nm}}$ & $=\frac{\substack{1000 \quad 2000 \\
\lambda, \operatorname{mm}_{1}}}{0.8}$ \\
\hline$r_{1}=160 \mathrm{~nm}, \lambda \max _{\mathrm{ext}}^{3000}=1450 \mathrm{~nm}$ & 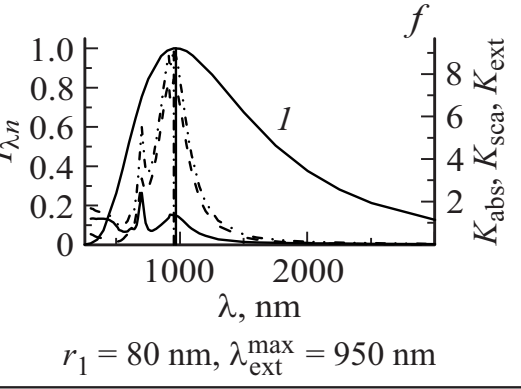 & $\begin{aligned} r_{1}=50 \mathrm{~nm}, \lambda_{\mathrm{ext}}^{\max _{2}}=750 \mathrm{~nm} \\
0.8 \\
0.4\end{aligned}$ \\
\hline$r_{1}=170 \mathrm{~nm}, \lambda_{\mathrm{ext}}^{\max }=1650 \mathrm{~nm}$ & $\begin{aligned} 1000 \underset{\lambda, \mathrm{nm}}{2000} \\
r_{1}=100 \mathrm{~nm}, \lambda \operatorname{maxt}_{\mathrm{ext}}=1070 \mathrm{~nm}\end{aligned}$ & $\begin{array}{c}1.0=0 \\
r_{1}=70 \mathrm{~nm}, \lambda \lambda_{\mathrm{ext}}^{\max }=892 \mathrm{~nm}\end{array}$ \\
\hline$=\underbrace{d}_{\substack{\lambda, \mathrm{nm} \\
r_{1}=130(1), 160(2), 170(3), \mathrm{nm}}}$ & $\begin{array}{c}\substack{2000 \quad 4000 \\
\lambda, \mathrm{nm}} \\
r_{1}=60(1), 80(2), 100(3), \mathrm{nm}\end{array}$ & 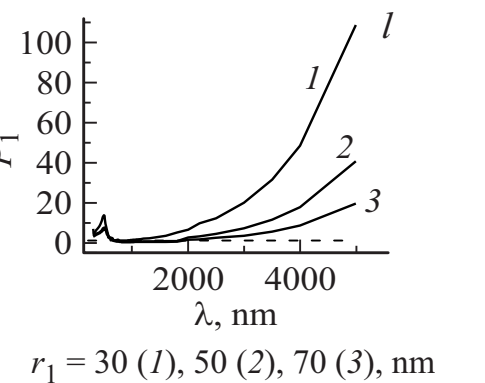 \\
\hline
\end{tabular}

Рис. 2. Зависимости факторов эффективности $K_{\mathrm{abs}}\left(a-c, e-g, i-k\right.$, сплошные линии), $K_{\mathrm{sca}}(a-c, e-g, i-k$, штриховые линии) и $K_{\mathrm{ext}}\left(a-c, e-g, i-k\right.$, штрихпунктирные линии), оптические интенсивности $I_{\lambda n}(\lambda)(a-c, e-g, i-k$, сплошные линии 1$)$ и параметpa $P_{1}(d, h, l)$ для $T_{S}=2000 \mathrm{~K}(a-d), 3000 \mathrm{~K}(e-h), 4000 \mathrm{~K}(i-l)$ от длины волны $\lambda$ для НЧ $\mathrm{SiO}_{2}-$ Аи с толщиной оболочки $\Delta r_{1}=10 \mathrm{~nm}(a-l)$ и радиусом $r_{1}=30(i, l 1), 50(j, l 2), 70(k, l 3), 60(e, h 1), 80(f, h 2), 100(g, h 3), 130(a, d 1), 160(b, d 2)$, $170(c, d 3) \mathrm{nm}$. Горизонтальные штриховые линии показывают значение $P_{1}=1(d, h, l)$. Представлены значения $r_{1}$ и $\lambda_{\mathrm{ext}}^{\max }$ для соответствующих зависимостей.

На рис. 3 приведены зависимости $K_{\text {abs }}$, $K_{\text {sca }}$ и $K_{\text {ext }}$ и $P_{1}$ от длины волны $\lambda$ для сферических двухслойных НЧ $\mathrm{Fe}_{3} \mathrm{O}_{4}-\mathrm{Au}$ с внешними радиусами $r_{1}=30-175 \mathrm{~nm}$ и толщиной оболочки $\Delta r_{1}=10 \mathrm{~nm}$. Следует отметить, что основные особенности упомянутых зависимостей оптических характеристик для $\mathrm{HЧ} \mathrm{SiO}_{2}-\mathrm{Au}$ с аналогичной толщиной оболочки существуют и в этом случае. Эти особенности можно сравнить, например, для значений $r_{1}=30,70,100 \mathrm{~nm}$ двух $\mathrm{HЧ} \mathrm{SiO}_{2}-\mathrm{Au}$ и $\mathrm{Fe}_{3} \mathrm{O}_{4}-\mathrm{Au}$. Для $\mathrm{HЧ} \mathrm{SiO}_{2}-\mathrm{Au}$ и $\mathrm{Fe}_{3} \mathrm{O}_{4}-\mathrm{Au}$ при $r_{1}=30,70,100 \mathrm{~nm}$ значения $K_{\mathrm{ext}}^{\max }$ располагаются соответственно в точках $\lambda_{\mathrm{ext}}^{\max }=620,892,1070 \mathrm{~nm}$ и в достаточно близких 


\begin{tabular}{|c|c|c|}
\hline \multicolumn{3}{|c|}{$\mathrm{Fe}_{3} \mathrm{O}_{4}-\mathrm{Au}, \Delta r_{1}=10 \mathrm{~nm}$} \\
\hline$T=2000 \mathrm{~K}, \lambda_{\max }=1450 \mathrm{~nm}$ & $T=3000 \mathrm{~K}, \lambda_{\max }=966 \mathrm{~nm}$ & $T=4000 \mathrm{~K}, \lambda_{\max }=724.5 \mathrm{~nm}$ \\
\hline$r_{1}=130 \mathrm{~nm}, \lambda_{\mathrm{ext}}^{\max }=1260 \mathrm{~nm}$ & $\frac{1000 \quad 2000}{\lambda, \mathrm{nm}_{0}^{1.0}}$ & $r_{1}=30 \mathrm{~nm}, \lambda_{\mathrm{ext}}^{\max }=620 \mathrm{~nm}$ \\
\hline$r_{1}=150 \mathrm{~nm}, \lambda_{\mathrm{ext}}^{\max }=1500 \mathrm{~nm}$ & 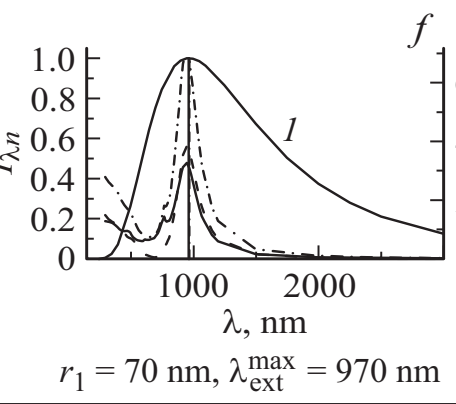 & $r_{1}=40 \mathrm{~nm}, \lambda_{\mathrm{ext}}^{\max }=750 \mathrm{~nm}$ \\
\hline$r_{1}=175 \mathrm{~nm}, \lambda_{\mathrm{ext}}^{\max }=1600 \mathrm{~nm}$ & 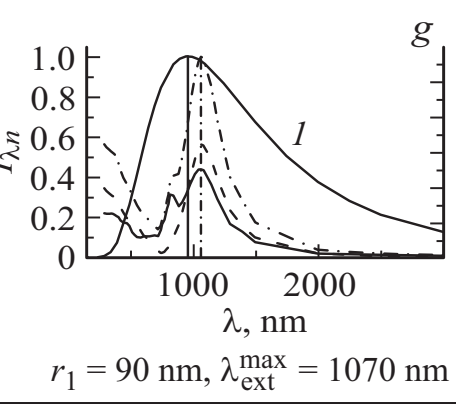 & $r_{1}=50 \mathrm{~nm}, \lambda_{\mathrm{ext}}^{\max }=850 \mathrm{~nm}$ \\
\hline$r_{1}=100(1), 150(2), 175(3), \mathrm{nm}$ & 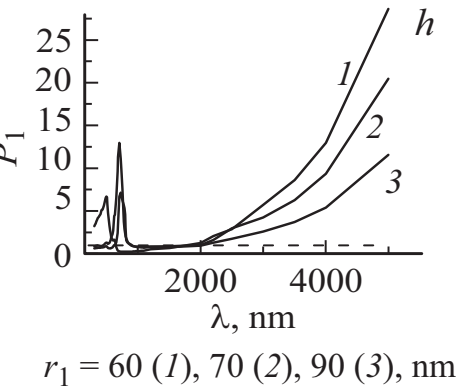 & $\begin{aligned} & 2000 \quad 4000 \\
& \lambda, \mathrm{nm} \\
& r_{1}=30(1), 40(2), 50(3), \mathrm{nm}\end{aligned}$ \\
\hline
\end{tabular}

Рис. 3. Зависимости факторов эффективности $K_{\mathrm{abs}}\left(a-c, e-g, i-k\right.$, сплошные линии), $K_{\mathrm{sca}}(a-c, e-g, i-k$, штриховые линии) и $K_{\mathrm{ext}}\left(a-c, e-g, i-k\right.$, штрихпунктирные линии), оптической интенсивности $I_{\lambda n}(\lambda)(a-c, e-g, i 0-k$, сплошные линии 1$)$ и параметра $P_{1}(d, h, l)$ для $T_{S}=2000 \mathrm{~K}(a-d), 3000 \mathrm{~K}(e-h), 4000 \mathrm{~K}(i-l)$ от длины волны $\lambda$ для НЧ $\mathrm{Fe}_{3} \mathrm{O}_{4}-\mathrm{Au} \mathrm{с} \mathrm{толщиной} \mathrm{оболочки}$ $\Delta r_{1}=10(a-l) \mathrm{nm}$ и радиусом $r_{1}=30(i, l 1), 40(j, l 2), 50(k, l 3), 60(e, h 1), 70(f, h 2), 90(g, h 3), 100(a, d 1), 150(b, d 2)$, $175(c, d 3) \mathrm{nm}$. Горизонтальные штриховые линии показывают значение $P_{1}=1(d, h, l)$. Представлены значения $r_{1}$ и $\lambda_{\mathrm{ext}}^{\max }$ для соответствующих зависимостей.

точках $\lambda_{\text {ext }}^{\max }=660,970,1150 \mathrm{~nm}$. Это свидетельствует об определенной близости зависимостей оптических характеристик для представленных НЧ. Максимальные значения $K_{\mathrm{ext}}^{\max }$ приблизительно равны 7 и 6 для НЧ $\mathrm{SiO}_{2}-\mathrm{Au}$ и $\mathrm{Fe}_{3} \mathrm{O}_{4}-\mathrm{Au}$ соответственно с $r_{1}=100 \mathrm{~nm}$.
Но существуют также и различия в зависимости оптических факторов представленных двух НЧ от $\lambda$. Формирование второго резонансного максимума НЧ $\mathrm{Fe}_{3} \mathrm{O}_{4}-\mathrm{Au}$ начинается при $r_{1} \geq 150 \mathrm{~nm}$ в спектральном интервале $200-400 \mathrm{~nm}$. Форма зависимости $K_{\mathrm{ext}}(\lambda)$ су- 
щественно не меняется при увеличении $r_{1}$, но ширина резонансной линии, в границах которой $K_{\text {ext }}$ имеет значение $K_{\text {ext }} \geq 0.5$, для $\mathrm{HЧ} \mathrm{Fe}_{3} \mathrm{O}_{4}-\mathrm{Au}$ значительно расширяется по оси $\lambda$ при увеличении $r_{1}$ по сравнению c $\mathrm{H} Y \mathrm{SiO}_{2}-\mathrm{Au}$.

Параметр $P_{1}$ имеет более сложное поведение в зависимости от $\lambda$ в данном случае. При этом для всех значений $r_{1}=30-175 \mathrm{~nm}$ параметр $P_{1}$ имеет максимум при $\lambda \sim 500-1000 \mathrm{~nm}$. При $r_{1}=30-50 \mathrm{~nm} P_{1}>1$ во всем интервале 700-5000 nm. Однако при $r_{1}=100-175 \mathrm{~nm}$ $P_{1}<1$ в интервале $\sim 700-2000 \mathrm{~nm}$, и только после этого $P_{1}$ увеличивается до 3-15 при увеличении $\lambda$ до $5000 \mathrm{~nm}$.

\section{Заключение}

Сильное поглощение и ослабление излучения черного тела НЧ для экранирования видимого и ИК излучения с непрерывным спектром в широком спектральном интервале 200-5000 nm представляет значительный научный и технический интерес. Для эффективного ослабления излучения сплошного спектра необходимо подбирать соответствующую структуру НЧ (однородная, ядрооболочка и т.д.), материалы (металл, оксид и т.д.), ядра и оболочки, их размеры (радиус ядра, толщина оболочки) с реализацией максимального значения $K_{\mathrm{ext}}^{\max }$, доминирующей роли поглощения НЧ над рассеянием при $K_{\text {abs }}>K_{\text {sca }}, P_{1}>1$ (или $P_{1} \gg 1$ ) и максимальной близости $K_{\text {ext }}(\lambda)$ НЧ к зависимости оптической интенсивности $I_{n \lambda}(\lambda)$. Выбор основывается на компромиссе при одновременной реализации указанных условий.

Систематическое исследование зависимостей факторов эффективности $K_{\text {ext }}, K_{\text {abs }}, K_{\text {sca }}$ и параметра $P_{1}$ от размеров $\mathrm{HЧ} \mathrm{Fe}_{3} \mathrm{O}_{4}-\mathrm{Au}$ и $\mathrm{SiO}_{2}-\mathrm{Au}$, длины волны излучения было проведено в настоящей работе как предпосылка для реализации потенциала НЧ ядро-оболочка для ослабления излучения с температурой поверхности излучателя в интервале $2000-4000 \mathrm{~K}$. Проведены исследования и анализ оптических характеристик указанных НЧ для длин волн излучения в спектральном интервале 200-6000 nm и в диапазоне радиусов НЧ $r_{1}=30-175 \mathrm{~nm}$ с толщиной оболочки $\Delta r_{1}=10 \mathrm{~nm}$ на основе компьютерного моделирования (рис. 2,3).

Трансформация оптических свойств и их общие и частные особенности с изменением параметров НЧ представлены на рис. 2,3 и позволяют оценить возможность использования НЧ для ослабления излучения. Увеличение $r_{1}$ приводит к смещению максимального ослабления (поглощения) $K_{\mathrm{ext}}^{\max }$ в сторону увеличения значений $\lambda$ в ИК область. Это означает возможность сопоставления зависимости $K_{\text {ext }}(\lambda)$ с зависимостью $I_{n \lambda}(\lambda)$ вплоть до их практического наложения друг на друга в энергетически важных областях спектра излучения.

Представленные на рис. 2,3 местоположения $\lambda_{\mathrm{ext}}^{\max }$ максимальных значений фактора $K_{\mathrm{ext}}^{\mathrm{max}}$ для конкретной НЧ и нескольких значений ее геометрических размеров немного отличаются от значений $\lambda_{\max }$ для приведенных значений $T_{S}=2000,3000,4000 \mathrm{~K}$. Однако данные значения можно связать с другими значениями $T_{S}$ излучения ЧТ, пересчитанными по закону Вина (3). Следовательно, показана возможность перестройки по длине волны спектров ослабления (поглощения, рассеяния) излучения НЧ путем изменения ее параметров.

Параметр $P_{1}$ можно использовать для определения типов НЧ преимущественно как поглотителей излучения при $P_{1}>1$. Главной особенностью для всех представленных зависимостей $P_{1}\left(\lambda, r_{1}\right)$ является уменьшение $P_{1}$ с увеличением $r_{1}$ для всего спектрального интервала $\lambda \sim 200-5000 \mathrm{~nm}$. Интересно отметить значения $P_{1} \geq 10-100$ для $\mathrm{HЧ} \mathrm{SiO}_{2}-\mathrm{Au}$ и $\mathrm{Fe}_{3} \mathrm{O}_{4}-\mathrm{Au}$ с $r_{1}=30-175 \mathrm{~nm}$ в избранных спектральных интервалах с $\lambda>600-2000 \mathrm{~nm}$.

Все это означает, что в целом представленные НЧ $\mathrm{SiO}_{2}-\mathrm{Au}$ и $\mathrm{Fe}_{3} \mathrm{O}_{4}-\mathrm{Au}$ являются хорошими ослабителями (поглотителями) излучения в диапазоне оптического спектра 200-5000 nm и могут применяться в качестве возможных кандидатов для эффективного ослабления оптического излучения высокотемпературных источников.

\section{Список литературы}

[1] Пожаровзрывозащита / Составители Сечин А.И., Кырмакова О.С. Томск: Изд-во Томского политехнического ун-та, 2015. $248 \mathrm{c}$.

[2] McGrattan K.B., Baum H.R., Hamins A. // Thermal Radiation from Large Pool Fires. National Institute of Standards and Technology. NISTIR report 6546, 2000.

[3] Boulet P., Parent GAcem. Z., Collin A., SéroGuillaume O. // Fire Safety J. 2011. V. 46. P. 21.

[4] Cumber P.S. // Fire Safety J. 2009. V. 44. P. 580.

[5] Fang J., Wang J., Tu R., Shang R., Zhang Y.-M., Wang J.-J. // Int. J. Thermal Sciences. 2018. V. 124. P. 338.

[6] Zhou L., Zeng D., Li D., Chaos M. // Fire Safety J. 2017. V. 89. P. 16.

[7] Zhou K., Wang X. // Int. J. Thermal Sciences. 2019. V. 138. P. 12.

[8] Naeem J., Mazari A.A., Havelka A. // Fibres \& Textiles in Eastern Europe. 2017. V. 25. P. 65.

[9] https://www.pilkington.com/

[10] Bohren C.F., Huffman D.R. Absorption and Scattering of Light by Small Particles. Wiley-Interscience, 1983. 660 p.

[11] Kreibig U., Vollmer M. Optical Properties of Metal Clusters. Berlin: Springer-Verlag, 1995. 532 p.

[12] Handbook of Nanoparticles / Ed. by Aliofkhazraei M. Berlin: Springer, 2016. $1440 \mathrm{p}$.

[13] Fundamentals of Nanoparticles. Classifications, Synthesis Methods, Properties, and Characterization / Ed. by Makhlouf A, Barhoum A. Amsterdam: Elsevier-Verlag, 2018. 666 p.

[14] Pustovalov V.K. Handbook of Nanoparticles / Ed. by Aliofkhazraei M, Heidelberg: Springer-Verlag, 2016. Part 31. P. 819.

[15] Tang Y, Vlahovic B. // Nanoscale Res. Lett. 2013. V. 8. P. 65.

[16] Astafyeva L., Pustovalov V., Fritzsche W. // NanoStructures \& Nano-Objects. 2017. V. 12. P. 57. 
[17] Pustovalov V., Astafyeva L., Jean B. // Nanotechnology. 2009. V. 20. P. 225105.

[18] Bardhan R., Grady N.K, Ali T., Halas N.J. // ACS Nano. 2010. V. 4. P. 6169.

[19] Ghazanfari L., Khosroshahi M.E. // Mat. Sci. Eng. C. 2014. V. 42. P. 185.

[20] Byers C., Zhang H., Swearer D., Yorulmaz M., Hoener B., Huang D. // Sci. Adv. 2015. V. 1. P. 1500988.

[21] Laaksonen K., Suomela S., Puisto S., Rostedt N., AlaNissila T., Nieminen R. // J. Opt. Soc. Am. B. 2013. V. 30. P. 338.

[22] Pustovalov V., Astafyeva L. // J. Nanomaterials. 2015. V. 5. Article ID 812617.

[23] Gorji T.B., Ranjbar A.A. // Renew Sustain Environ Rev. 2017. V. 72. P. 10

[24] Pustovalov V.K. // Springer Nature Applied Sciences. 2019. V. 1. P. 356.

[25] Born M., Wolf E. Principles of Optics. Oxford: Pergamon Press. 1964.

[26] Refractive index database. http://refractiveindex.info/.

[27] Handbook of Optical Constants of Solids / Ed. by Palik E.D. Academic Press: N. Y. 